\title{
Optical measurement of cable and string vibration
}

\author{
Y. Achkire ${ }^{\mathrm{a}}$ and A. Preumont ${ }^{\mathrm{b}}$

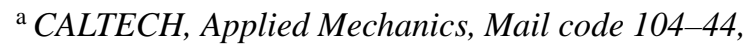 \\ Pasadena, CA 91125, USA \\ ${ }^{\mathrm{b}}$ Université Libre de Bruxelles, Active Structures \\ Laboratory, Department of Mechanical Engineering \\ and Robotics, CP 165, 50 Av. F.D. Roosevelt, 1050 \\ Brussels, Belgium
}

Received 7 January 1998

Revised 5 August 1998

This paper describes a non contacting measurement technique for the transverse vibration of small cables and strings using an analog position sensing detector. On the one hand, the sensor is used to monitor the cable vibrations of a small scale mock-up of a cable structure in order to validate the nonlinear cable dynamics model. On the other hand, the optical sensor is used to evaluate the performance of an active tendon control algorithm with guaranteed stability properties. It is demonstrated experimentally, that a force feedback control law based on a collocated force sensor measuring the tension in the cable is feasible and provides active damping in the cable.

Keywords: Photodetector, laser, cable vibration, active control, cable-stayed bridges

\section{Introduction}

Cable vibration has become a major issue in the design of cable stayed bridges, as their ever increasing span makes them more sensitive to flutter instability and to wind and traffic-induced vibrations. The problem is difficult because of the highly nonlinear behavior of cables with sag and the strong coupling between the cables and the bridge deck dynamics: the cables excite the bridge through the time varying tension and the deck excites the cables through the linear coupling (inertia) and the quadratic coupling terms; the latter may produce parametric excitation if some tuning conditions are satisfied [8,9].

Theoretical investigations of the active tendon control for flutter have been performed by Yang and Gi- annopoulos [12,13] and for string and cable vibrations by Chen [3] and Fujino et al. [4]; some experiments on small scale mock-ups have been reported [10,11]. The present authors [2] have established that, if one uses collocated actuator-sensor pairs, it is possible to develop a control algorithm with guaranteed stability. This was confirmed by experiments on a small scale mock-up involving a $2 \mathrm{~m}$ long cable of $0.5 \mathrm{~mm}$ diameter. In this context, an optical non contacting measurement system for cable and string vibrations has been developed.

\section{Sensing cable and string vibrations}

Cable vibrations can be measured through the variation of the tension in the cable; however, this signal includes a complex mixture of linear and quadratic terms; the former appears at the natural frequency of the cable while the latter appears at twice the natural frequency. Besides, the anti-symmetric modes are not linearly observable from the tension in the cable because their corresponding mode shapes do not produce any variation of the axial load in the cable. A direct measurement of the cable displacement can be obtained with a CCD camera [10] or with an analog position sensing detector (PSD) [5]. We have selected the PSD technology, which supplies analog signals proportional to the position of a light spot on the detector. The technology exists in one or two dimensions; it is linear, wide band, and perfectly adapted to the range of amplitudes of our application (typically cable vibrations of $10 \mathrm{~mm}$ ).

\section{Position sensing detector}

Analog position sensing photodetectors [6] use the lateral photoelectric effect to produce output signals proportional to the true $x$ and $y$ coordinates of the centroid of the light spot on the detector. Current carriers generated in the illuminated region on the detector are 


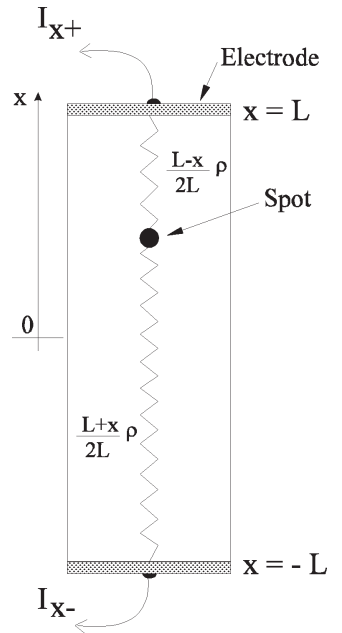

Fig. 1. Model of a one dimensional detector.

proportional to the intensity of the light spot and go to the electrodes in proportion to the conductance of the current paths between the illuminated region and the electrodes. Since the conductance of a current path is a function of the distance between the light spot and the corresponding electrode, the position information can be extracted by comparing the currents that reach the electrodes. For a one-dimensional detector (Fig. 1), the distance between the centroid of the light spot and the centre of the detector is given by

$$
x=L \frac{I_{x+}-I_{x-}}{I_{x+}+I_{x-}},
$$

where $I_{x+}$ and $I_{x-}$ are the currents measured at the two electrodes; this result is independent of the intensity of the light source. Although the PSD normally senses the centroid of a light spot, it can also sense a dark spot on a bright background, and this is the way it is used in our application.

\section{Optical system}

The principle of the optical measurement system is represented in Fig. 2; the light source is a $5 \mathrm{~mW}$ laser diode; its beam is expanded into a flat parallel beam by two cylindrical lenses; the cable is placed in such a way that the chord line is normal to the light plane. The light plane produces a line on the PSD, with a dark spot corresponding to the shade of the cable (Fig. 2). In order to improve the sensor signal, the PSD can be supplemented with a bandpass filter centered on the wavelength of the laser diode $(670 \mathrm{~nm}$ in this case).

The position of the centroid of the dark spot is given by

$$
x=L\left(1-\frac{L}{2 a}\right) \frac{I_{x+}-I_{x-}}{I_{x+}+I_{x-}},
$$

where $L$ is the length of the sensor and $a$ is the size of the dark spot.

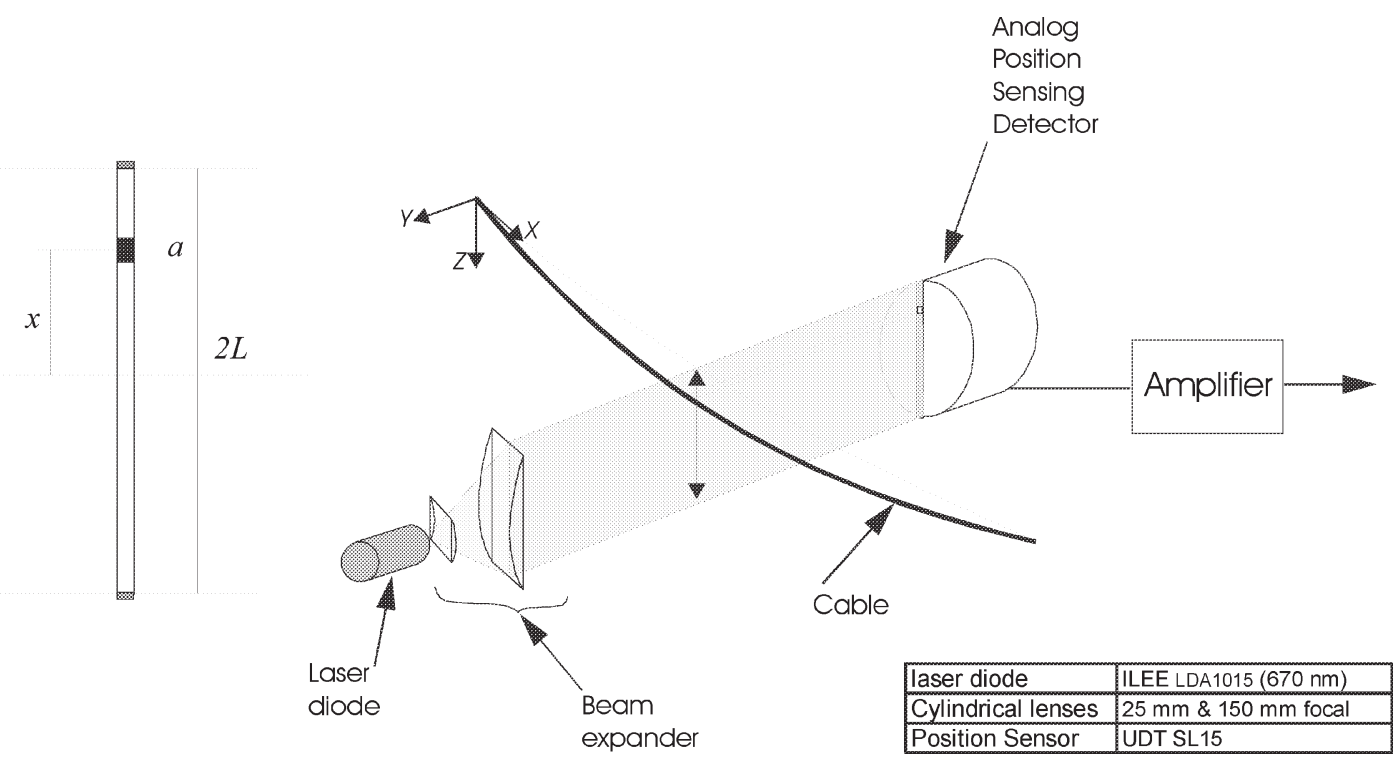

Fig. 2. Sensing vibrations of a wire. 


\section{Calibration}

Figure 3 describes the set-up used to calibrate the proposed measurement system by comparison with the output of a laser interferometer. A small cylindrical target simulating the cable and the target mirror of the interferometer are mounted on an axially movable common support induced by a shaker. A band-limited white noise is applied to the shaker input. The displacement of the cylindrical target induced by the axial motion of the shaker is measured using the optical device involving the laser diode, the two cylindrical lenses and the position sensing photodetector as indicated in Fig. 3 . Also, the axial displacement of the target mirror (i.e., the axial displacement of the shaker) is simultaneously and accurately measured by the laser interferometer.

The frequency response function between the interferometer and the PSD analog output has been measured for several values of the diameter of the cylindrical target $a$. The results are shown in Fig. 4; the

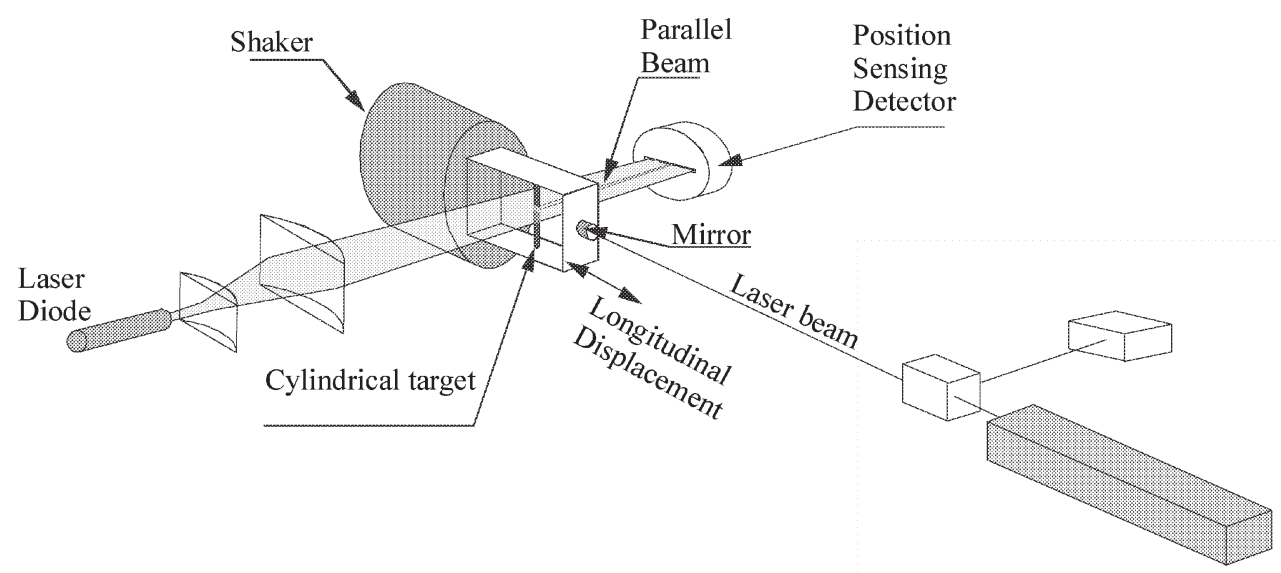

Laser interferometer device

Fig. 3. Calibration of the PSD device.
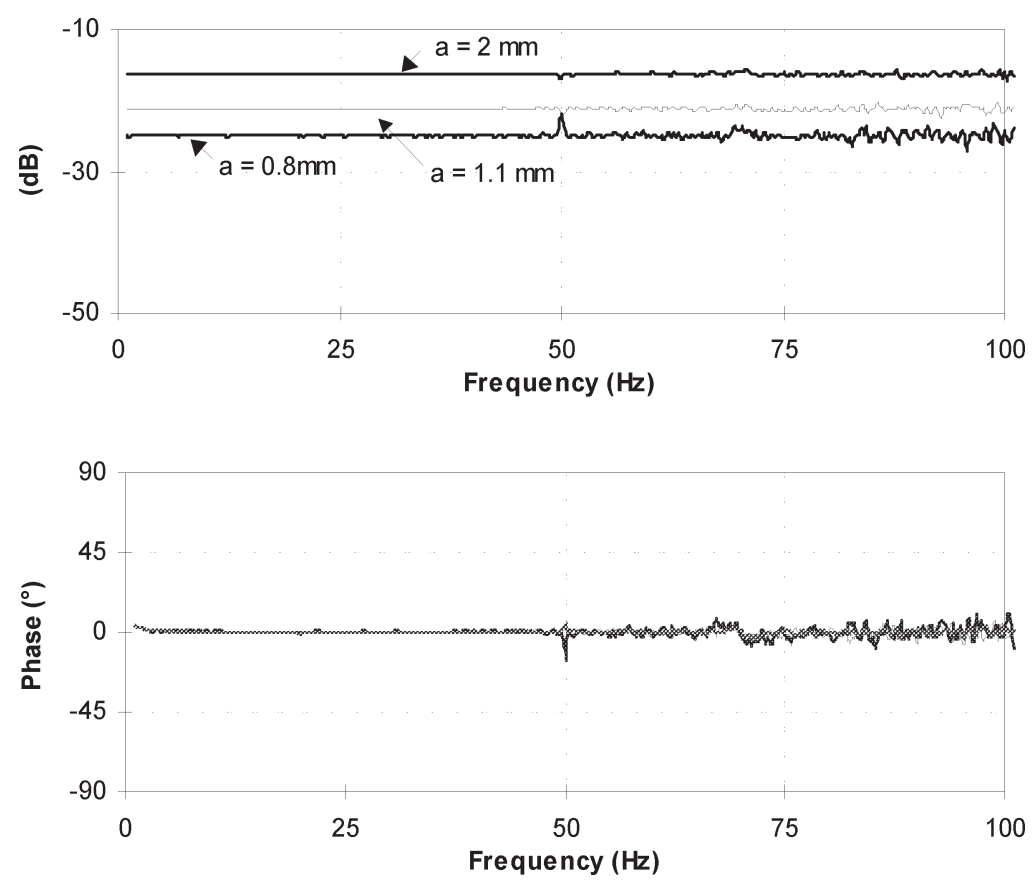

Fig. 4. Frequency response function between the laser interferometer displacement signal and the PSD output measurement $(0-100 \mathrm{~Hz})$. 

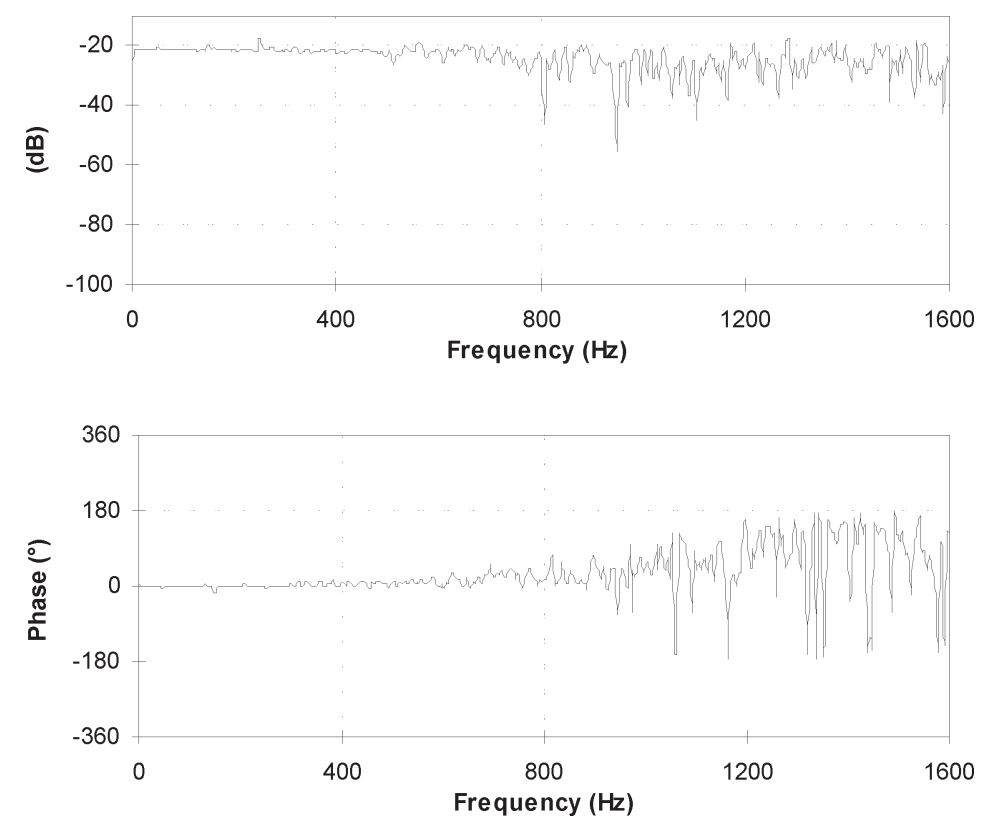

Fig. 5. Frequency response function between the laser interferometer position signal and the PSD output measurement $(0-1600 \mathrm{~Hz})$.

frequency response function is close to perfect at low frequency, with an extremely small phase lag; this makes this sensor very attractive for control applications. Experimental results also confirm the applicability of Eq. (2). Figure 5 shows the same frequency response function in the range $0-1600 \mathrm{~Hz}$.

In order to evaluate the accuracy of the position sensor, the output of the photodetector has been compared to the displacement measurement from the laser interferometer, when a harmonic signal at $20 \mathrm{~Hz}$ is applied to the shaker (Fig. 6); the accuracy is about $\pm 10 \mu \mathrm{m}$, it is mainly associated with noise in the amplifier.

\section{Experimental set-up}

As already mentioned in the introduction, this sensor has been developed to investigate the performance of an active tendon for the control of cable-structure systems [2,9]. The cable vibration alone has been investigated with the experimental set-up of Fig. 7. The cable is a $2 \mathrm{~m}$ long stainless steel wire of $0.196 \mathrm{~mm}^{2}$ cross section, provided with additional lumped masses at regular intervals, in order to achieve a representative value of the sag to span ratio. The mass per unit length is $0.057 \mathrm{~kg} / \mathrm{m}$ and the static tension in the cable is selected between $25 \mathrm{~N}$ and $50 \mathrm{~N}$ (this is done with the help of an adjustable weight, as indicated in Fig. 7. For a tension of $25 \mathrm{~N}$, the sag to span ratio is $0.5 \%$ and the first in-plane frequency is $5.7 \mathrm{~Hz}$. One end of the cable is fixed to a lever system which is used to amplify the motion of the active element, which consists of a piezoelectric linear actuator collocated with a force sensor (because of the high-pass behavior of the piezoelectric force sensor, it measures only the dynamic component of the tension in the cable). The amplification ratio of the lever arm is 3.4 , corresponding to a maximum axial displacement of $150 \mu \mathrm{m}$ for the moving support. The controller is implemented digitally on a DSP board. An electrodynamic shaker equipped with a force sensor is placed near the other end of the cable to generate the in-plane excitation and the new optical sensor is installed as indicated in the figure, in order to measure the in-plane cable vibrations.

\section{Governing equations}

We propose a non-linear model of the inclined cable which accounts for coupling between the in-plane and out-of-plane motions, and also for the displacement of the support point (see Fig. 8); this problem has been considered in $[7,10]$. The model of the cable is written in a local coordinate system as indicated in Fig. 8; the local $x$ axis is taken along the chord line while the $z$ axis is in the gravity plane and perpendicular to the chord line. The equations governing the motion, ex- 
Y. Achkire and A. Preumont / Optical measurement of cable and string vibration

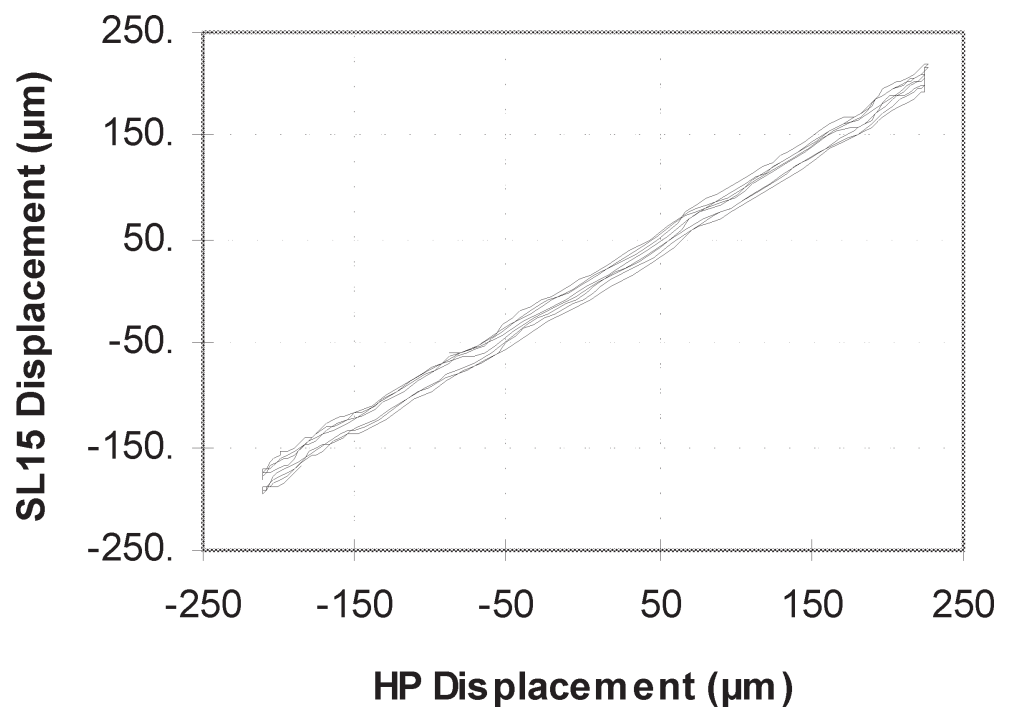

Fig. 6. Displacement measured by the photodetector vs HP laser interferometer.

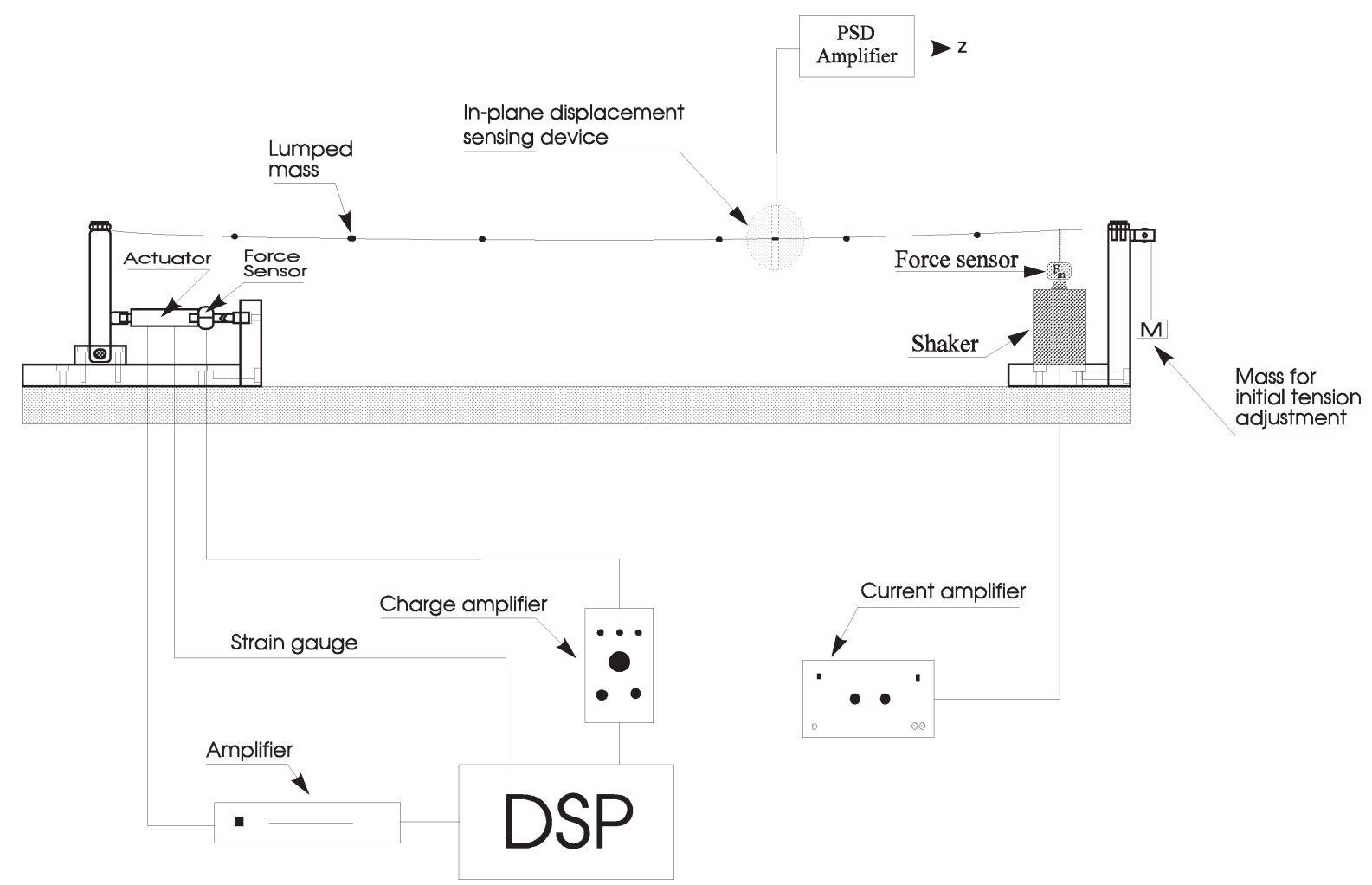

Fig. 7. Cable test set-up. 


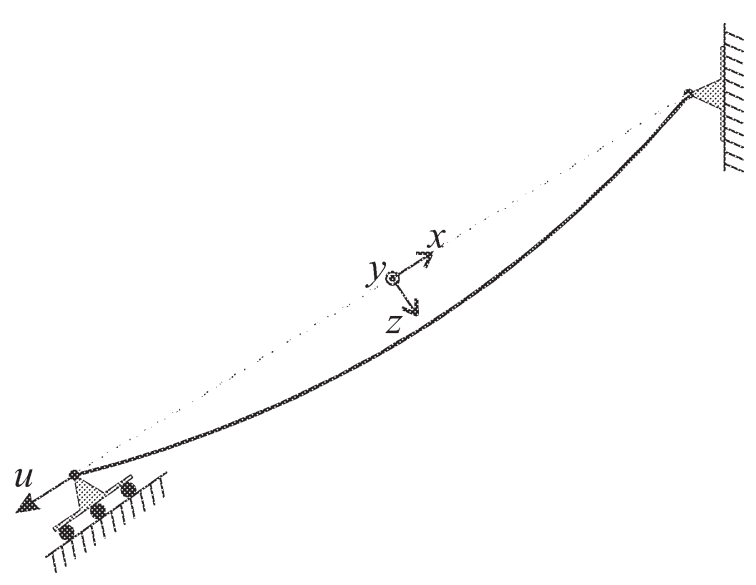

Fig. 8. Cable model.

pressed in modal coordinates have been derived and their detailed development can be found in [1],

Out of plane:

$$
\begin{gathered}
m_{y n}\left(\ddot{y}_{n}+2 \xi_{y n} \omega_{y n} \dot{y}_{n}+\omega_{y n}^{2} y_{n}\right) \\
+\underbrace{\sum_{k} \nu_{n k} y_{n}\left(y_{k}^{2}+z_{k}^{2}\right)}_{\text {cable stretching }} \\
+\underbrace{\sum_{k} 2 \beta_{n k} y_{n} z_{k}}_{\text {cable sag }} \\
+\underbrace{R_{n} u y_{n}}_{\text {active stiffness variation }}=F_{y n} .
\end{gathered}
$$

In plane:

$$
\begin{aligned}
& m_{z n}\left(\ddot{z}_{n}+2 \xi_{z n} \omega_{z n} \dot{z}_{n}+\omega_{z n}^{2} z_{n}\right) \\
& +\underbrace{\sum_{k} \nu_{n k} z_{n}\left(y_{k}^{2}+z_{k}^{2}\right)}_{\text {cable stretching }} \\
& +\underbrace{\sum_{k} 2 \beta_{n k} z_{n} z_{k}}_{\text {cable sag }}+\underbrace{R_{n} u z_{n}}_{\text {active stiffness variation }} \\
& +\underbrace{\sum_{k} \beta_{k n}\left(y_{k}^{2}+z_{k}^{2}\right)}_{\text {cable sag }} \\
& -\underbrace{\text { active sag induced force }}_{\alpha_{n} \frac{\mathrm{d}^{2} u}{\mathrm{~d} t^{2}}}=F_{z n} .
\end{aligned}
$$

Tension in the cable:

$$
\begin{array}{r}
T=\underbrace{h_{u} u}_{\text {feedthrough }}+\underbrace{\sum_{n} h_{1 n} z_{n}}_{\text {cable sag }} \\
+\underbrace{\sum_{n} h_{2 n}\left(y_{n}^{2}+z_{n}^{2}\right)}_{\text {cable stretching }},
\end{array}
$$

where $y_{n}$ and $z_{n}$ refer to the modal amplitudes of the in-plane and out-of-plane modes respectively, and $u$ represents the axial motion of the support. $F_{y n}$ and $F_{z n}$ represent the modal forces applied to the cable. In Eq. (5) $h_{u}$ is the static stiffness of the cable. In addition to the tendon force $h_{u} u$, the dynamical tension $T$ has a linear and a quadratic contribution; the former is due to the presence of cable sag and includes the in-plane symmetric modes only, because $h_{1 n}=0$ if $n$ is even; the latter is due to cable stretching. The analytical form of the coefficients can be found in reference [11]. The physical meaning of the various terms is indicated in the equations. Notice that:

- The active sag induced force appears only in the equations governing the in-plane motion. In addition, $\alpha_{n}=0$ and $h_{1 n}=0$ if $n$ is even (i.e., for antisymmetric modes).

- The out-of-plane modes affect the tension in the cable only with the quadratic term (corresponding to cable stretching), while the in-plane symmetric modes have a linear influence on $T$.

Also we can see (Eqs (3), (4)) that the nonlinearities of the cable dynamics appear through the quadratic and cubic couplings between modes and through the parametric excitation; the latter is induced by the differential motion of the cable supports (terms $R_{n u} y_{n}$ and $R_{n u} z_{n}$ ) which exist for both in-plane and out-of-plane modes.

\section{Verification of the cable model}

Figure 9(a) shows the frequency response function between the displacement $u$ of the active tendon and the in-plane vibration of the cable (amplitude $z$ ); we see that all the modes of odd number $(1,3,5)$ are excited through the active sag induced force (see Eq. (4)), the modes of even number $(2,4)$ are not excited because the corresponding coefficients $\alpha_{n}=0$. The contributions at $2 \omega_{1}, 2 \omega_{2}$ correspond to the parametric res- 

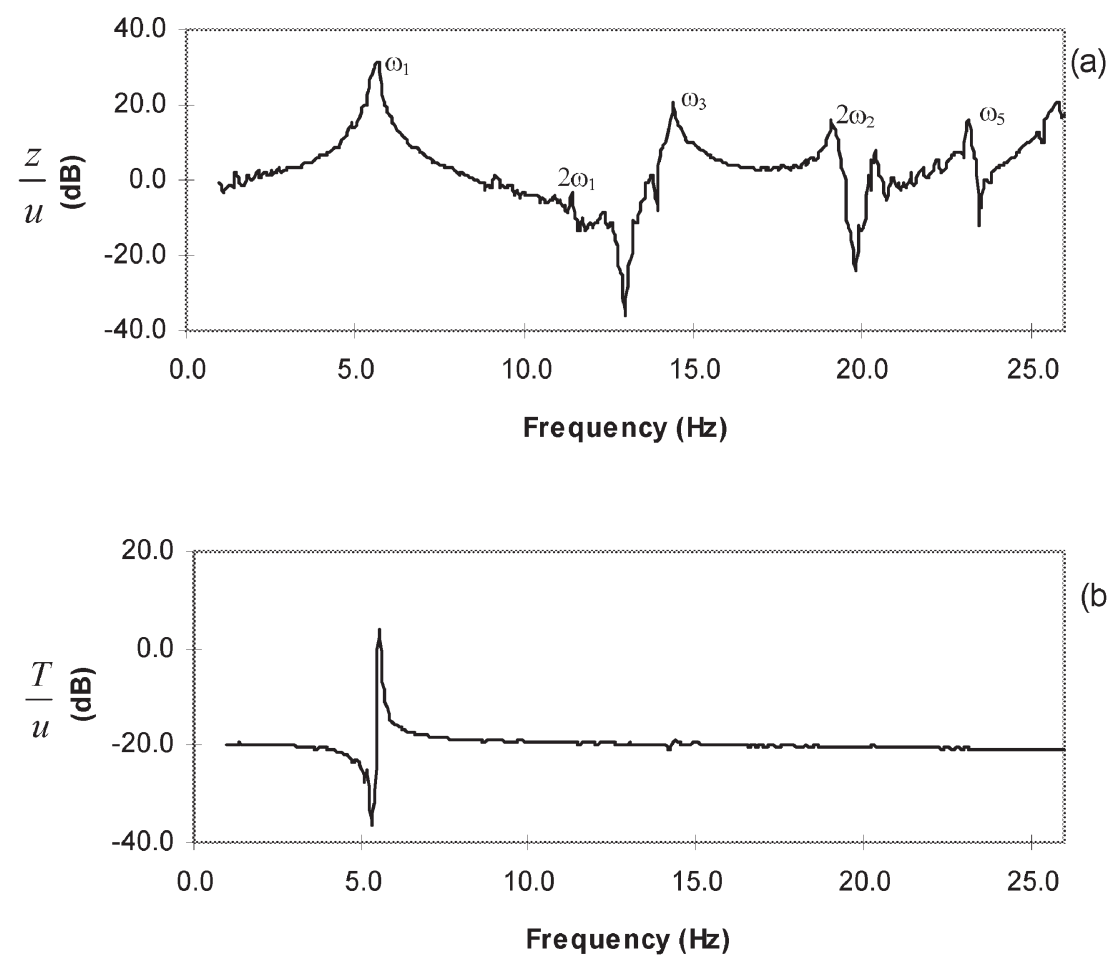

(b)

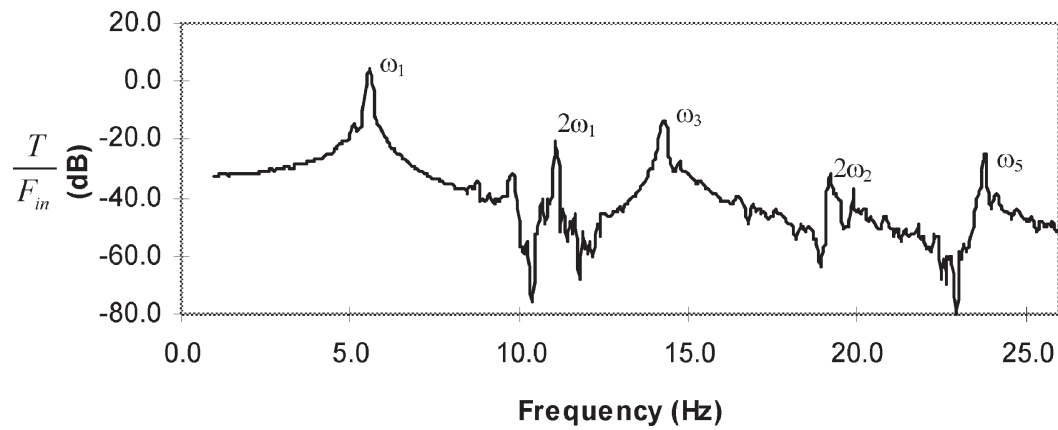

(c)

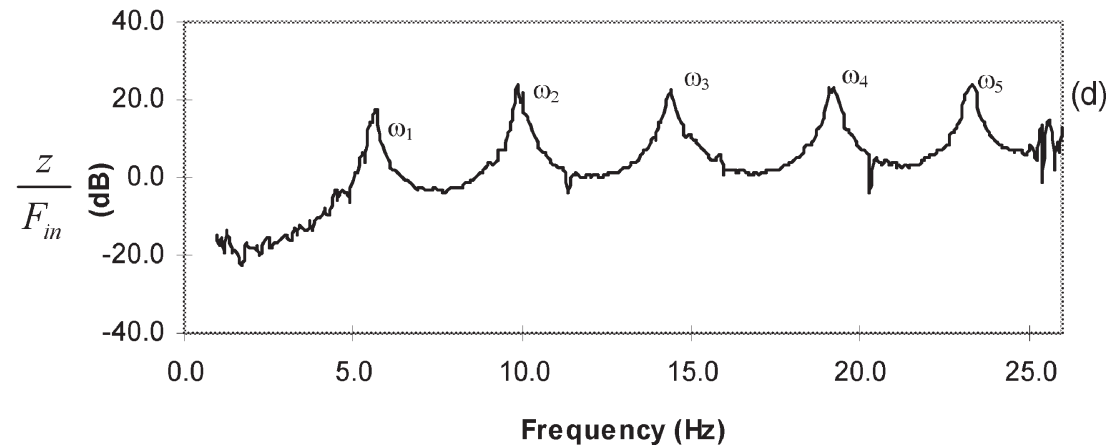

Fig. 9. Frequency response function between: (a) the active tendon displacement $u$ and the amplitude $z$ of the in-plane vibration; (b) the active tendon displacement $u$ and the tension $T$ in the cable; (c) the shaker force $F_{i n}$ and the tension $T$ in the cable; (d) the shaker force $F_{\text {in }}$ and the amplitude $z$ of the in-plane vibration of the cable. 


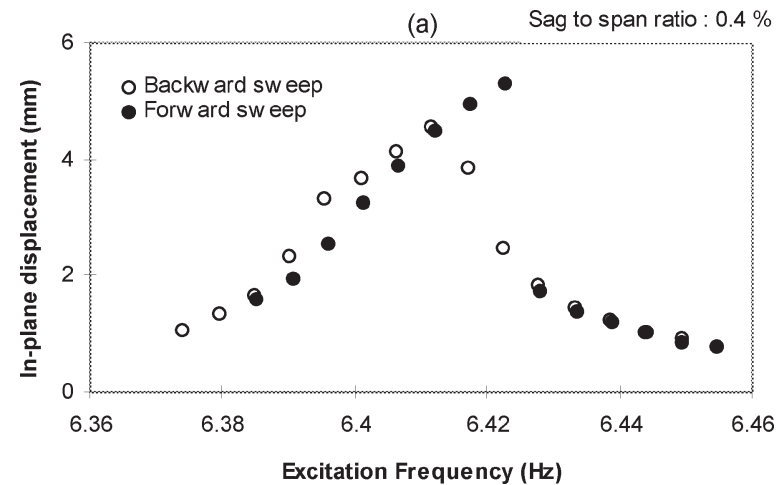

(b)

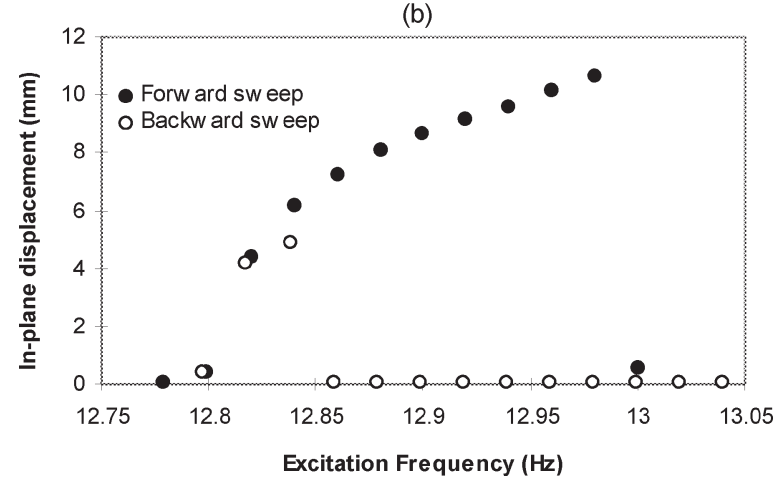

Fig. 10. Frequency response function between the active tendon displacement and the in-plane vibration at (a) the primary resonance $\omega_{1}$, and (b) the parametric resonance $2 \omega_{1}$.

onance; we observe that the parametric resonance of mode 2 is much more pronounced than that of mode 1 . Figure 9(b) shows the frequency response function between the active tendon and the tension in the cable (i.e., the open-loop transfer function of the control system); we see that it is dominated by the first mode and that there is a transmission zero right before $\omega_{1}$. Figure 9(c) shows the frequency response between the shaker force $F_{\text {in }}$ and the tension in the cable; we see that mode 2 appears only through the quadratic term at $2 \omega_{2}$, because the coefficients $h_{1 n}=0$ for $n$ even in Eq. (5); by contrast, mode 1 appears at $\omega_{1}$, with the linear term and at $2 \omega_{1}$, with the quadratic term in Eq. (5). Figure 9(d) shows the frequency response between the shaker force and the in-plane vibration; all the modes contribute to the response as we would expect from Eq. (4). All the above frequency responses have been obtained with a white noise excitation.

Figure 10(a) shows the frequency response function between the active tendon displacement and the in-plane vibration in the vicinity of the primary resonance, when the input excitation is a slow sine sweep (each frequency point corresponds to a steady state

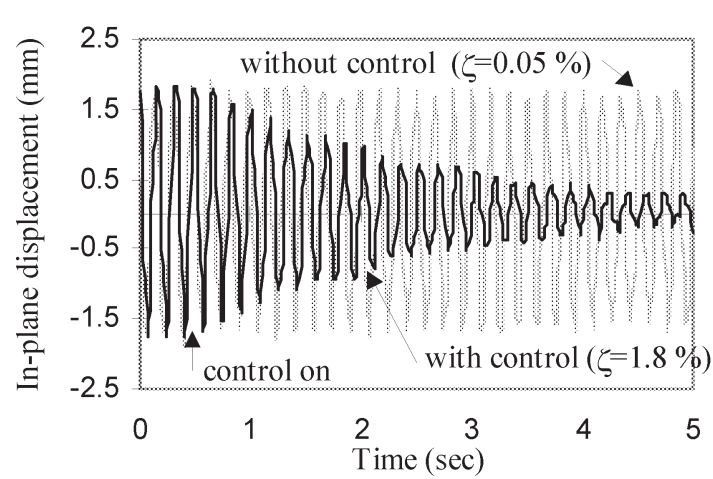

(a)

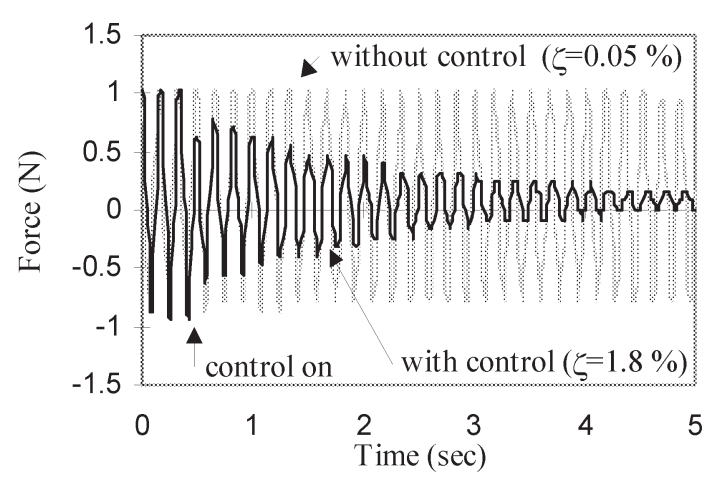

(b)

Fig. 11. Effect of the Integral Force Feedback on the free oscillations of the cable.

harmonic excitation of $3 \mathrm{~min}$ ); we notice clearly the jump phenomenon associated with the stiffening effect of the cubic nonlinearity in Eq. (4) (cable stretching). Figure 10(b) shows the same curve near the parametric resonance, in the vicinity of $2 \omega_{1}$; the jump phenomenon is even more pronounced. Similar results have been obtained by Nayfeh et al. [8].

\section{Active control}

The use of the active tendon for the damping of cable structures has been described in previous papers [2,9]; it has been shown that, when the actuator and the force sensor are collocated, the Integral Force Feedback law relating the actuator displacement $u$ to the dynamical component of the tension $T$ by,

$$
u=g \int T \mathrm{~d} t
$$

has guaranteed stability (assuming perfect actuator and sensor dynamics) because it produces an energy absorbing control. Note that, for positive gain $g$ the power 
flow from the proposed control system $W=-T \dot{u}=$ $-g T^{2}$ is negative. This strategy has been successfully demonstrated experimentally for cable-structures; the control law is indeed always stable, even at the parametric resonance, when the frequency of the structure is exactly twice that of the cable. Figure 11 shows the effect of the control strategy on a cable alone; with reference to Eq. (4), the control acts through the active sag induced forces, which depend on the sag in the system; the control works better when the sag increases. Figure 11(a) shows the free response of the vibration amplitude (in-plane displacement of the cable), with and without control; we see that, even for this small value of the sag to span ratio $(0.4 \%)$, the active damping is substantial ( $\xi=1.8 \%$ instead of $0.05 \%)$. These results are confirmed by Fig. 11(b), which shows the dynamic tension in the cable. Note that there is a sudden jump in the force diagram when the control is applied, as a result of the feedthrough component in the tension Eq. (5).

\section{Conclusion}

A non-contacting optical measurement system has been developed for the transverse vibration of small cables and strings. A flat parallel laser beam illuminates the vibrating cable and an analog position sensing photodetector measures the displacement of the shade created by the cable. The stroke of the sensor is $\pm 10 \mathrm{~mm}$ and the accuracy is $\pm 10 \mu \mathrm{m}$ in the frequency range $0-100 \mathrm{~Hz}$. The sensor has been used to evaluate the performances of a control system based on the use of an active tendon for the damping of cable structures. The guaranteed stability and damping properties of the proposed control strategy (Integral Force Feedback) have been demonstrated experimentally on a lab- oratory mock-up. Active damping of $1.8 \%$ has been achieved for cables with small sag, which is superior to previously published results.

\section{References}

[1] Y. Achkire, Active tendon control of cable-stayed bridges. Ph.D. thesis, Free University of Brussels, 1997.

[2] Y. Achkire and A. Preumont, Active tendon control of cablestayed bridges, Earthquake Engrg. Struct. Dyn. 25(6) (1996), 585-597.

[3] J-C. Chen, Response of large space structures with stiffness control, AIAA, J. Spacecraft 21(5) (1984), 463-467.

[4] Y. Fujino, P. Warnitchai and B.M. Pacheco, Active stiffness control of cable vibration, Trans. ASME, J. Appl. Mech. 60 (1993), 948-953.

[5] C.L. Lee and N.C. Perkins, Experimental investigation of isolated and simultaneous internal resonances in suspended cables, J. Vibration and Acoustics 117 (1995), 385-391.

[6] L.E. Lindholm and I.K. Edwards, Analog position-sensing photodetectors, Photonics Spectra, November 1991.

[7] A.H. Nayfeh and D.T. Mook, Non Linear Oscillations, Wiley, New York, 1979.

[8] S.A. Nayfeh, A.H. Nayfeh and D.T. Mook, Nonlinear response of a taut string to longitudinal and transverse end excitation, J. Vibration and Control 1 (1995), 307-334.

[9] A. Preumont and Y. Achkire, Active damping of structures with guy cables, AIAA J. Guidance, Dynamics and Control 20(2) (1997), 320-326.

[10] T. Susumpow and Y. Fujino, An experimental study on active control of planar cable vibration by axial support motion, Earthq. Engrg. Struct. Dyn. 23 (1994), 1283-1297.

[11] P. Warnitchai, Y. Fujino, B.M. Pacheco and R. Agret, An Experimental study on active tendon control of cable-stayed bridges, Earthq. Engrg. and Struct. Dyn. 22(2) (1993), 93-111.

[12] J.N. Yang and F. Giannopoulos, Active control and stability of cable-stayed bridge, ASCE, J. Eng. Mech. Div. 105 (1979), 677-694.

[13] J.N. Yang and F. Giannopoulos, Active control of two-cablestayed bridge, ASCE, J. Eng. Mech. Div. 105 (1979), 795-810. 

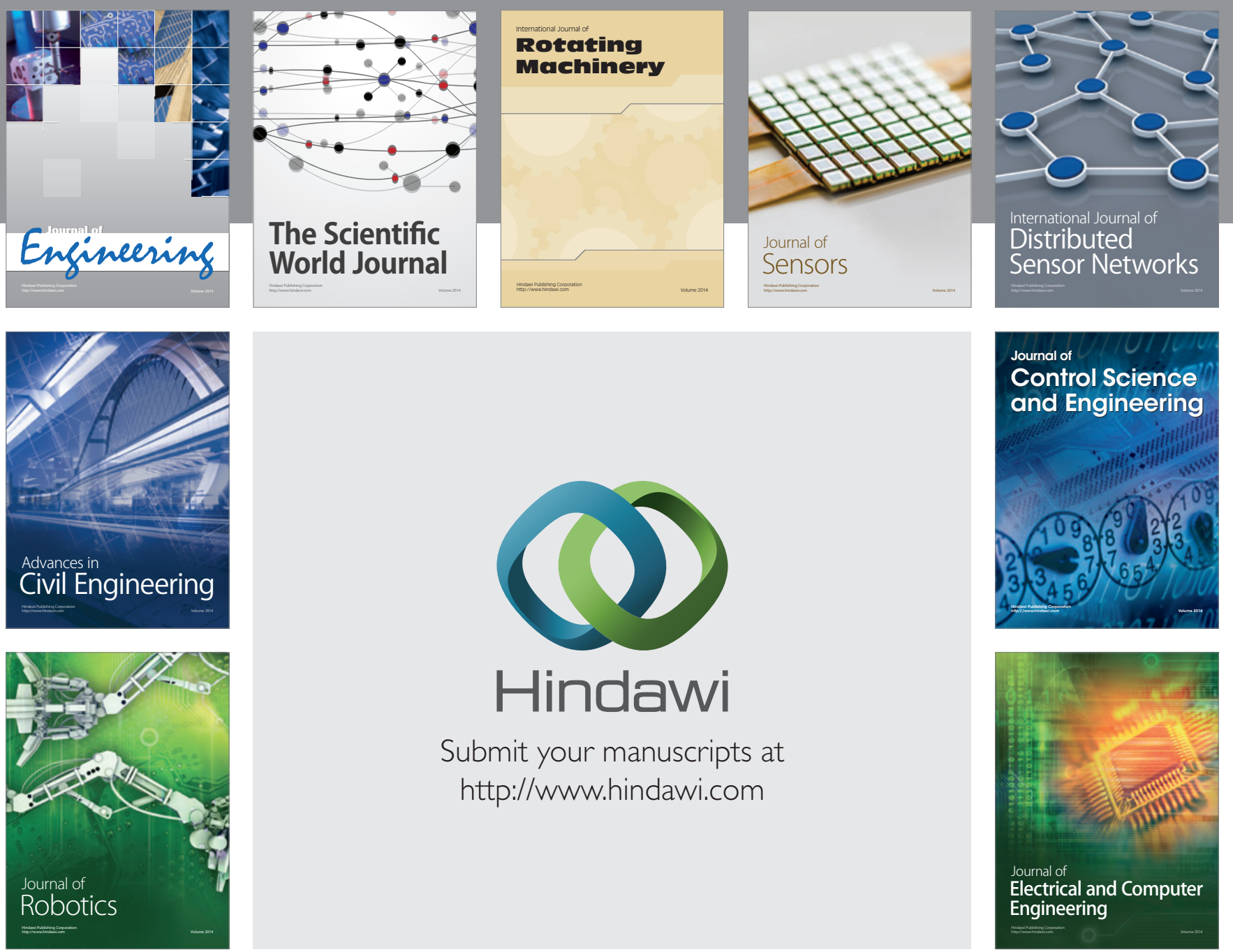

Submit your manuscripts at

http://www.hindawi.com
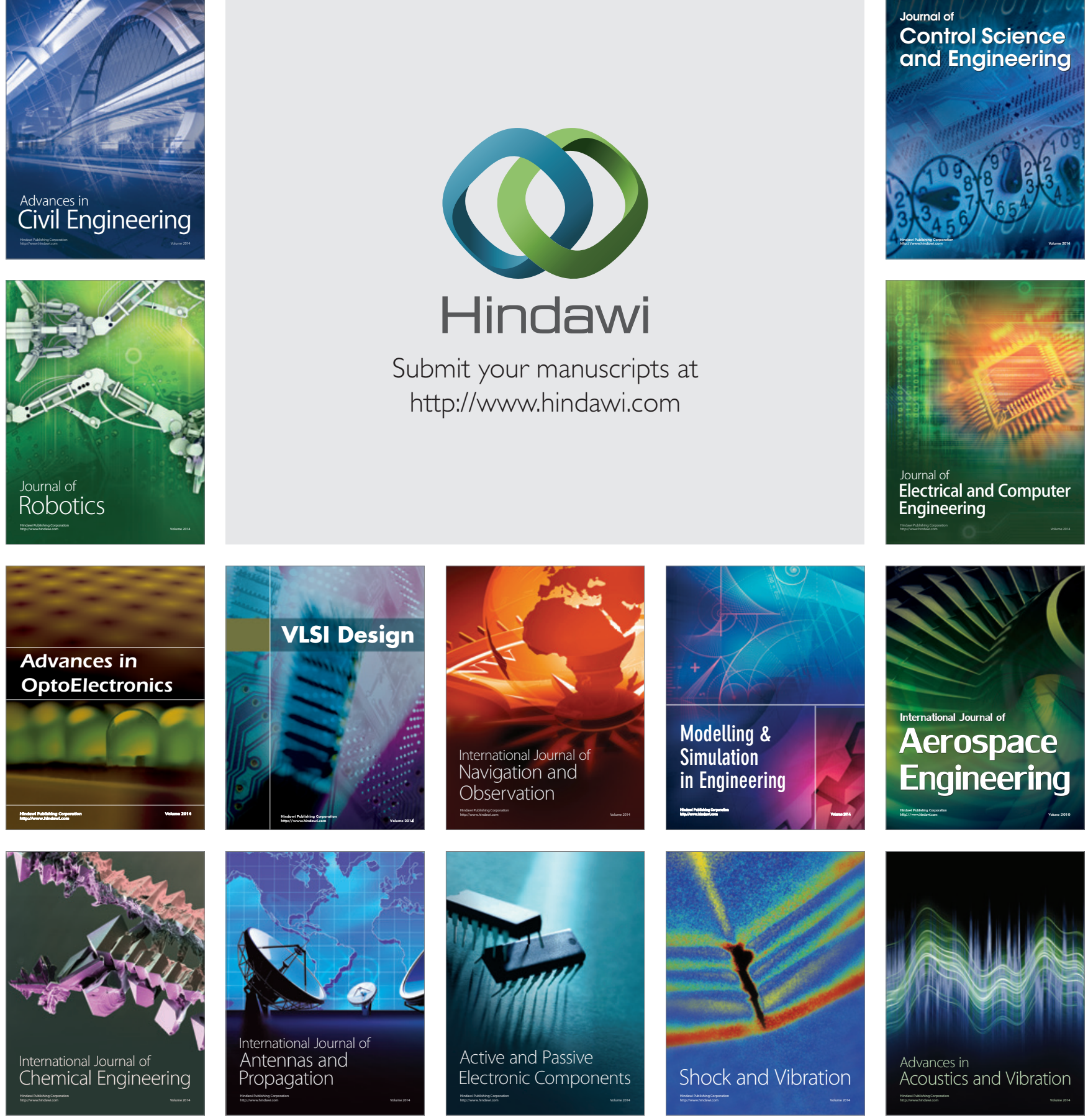